\title{
NEUTRAL HYDROGEN AT LARGE DISTANCES \\ FROM PARENT GALAXIES
}

\author{
R. D. DA VIES \\ University of Manchester, Nuffield Radio Astronomy Laboratories, Jodrell Bank, U.K.
}

\begin{abstract}
High sensitivity measurements in the vicinity of nearby galaxies have shown the existence of appreciable quantities of neutral hydrogen at large distances ( 2 to 5 Holmberg radii) from the centre. The galaxies studied were the M81/M82/NGC 3077 system, M31, M33, IC 342 and M51. In all cases the gas is at velocities consistent with being bound to the parent galaxy. Of these the M81 system contains the most circum-galactic neutral hydrogen. In several galaxies there is as much neutral hydrogen outside the Holmberg dimensions as inside.
\end{abstract}

\section{Introduction}

Neutral hydrogen surveys of nearby spiral galaxies show that the main neutral hydrogen concentrations lie in the regions of the well-developed spiral arms. Both pencil-beam and aperture synthesis observations place the bulk of the neutral gas within the galactic dimensions given by Holmberg (1958). There are, however, plausible reasons why neutral hydrogen may be more broadly distributed than the luminosity in a galaxy:

(a) Gas may lie in parts of the disk where the density is too low to allow star formation, a situation already known to exist in the Sd galaxy NGC 6822. This could be primordial gas with high angular momentum.

(b) Gas may be falling into a galaxy, either from intergalactic space or after ejection from the active nucleus of the galaxy itself (Oort, 1969).

(c) The gas may be intracluster gas which is gravitationally bound to one of the cluster galaxies.

(d) The gas may be torn from the inner spiral arms by the tidal interaction with a neighbouring galaxy. Tails and bridges which are formed in this way can lie far outside the main spiral structure (e.g. Toomre and Toomre, 1972).

The results described here bring together evidence obtained at Jodrell Bank for neutral hydrogen outside the optical confines of some nearby spiral galaxies.

\section{The Observing Techniques}

All the observations were made using a beam-switching technique with the Mark I $250 \mathrm{ft}$ radio telescope, the system temperature being about $125 \mathrm{~K}$. The signals were processed by a 256 channel autocorrelation receiver having an overall bandwidth of $5.0 \mathrm{MHz}$, which corresponds to a velocity range of $1060 \mathrm{~km} \mathrm{~s}^{-1}$, and a velocity resolution of $7 \mathrm{~km} \mathrm{~s}^{-1}$. Observations of IC 342 were taken with the Mark I radio telescope which was illuminated to give a beamwidth of $13^{\prime} \times 17^{\prime}$; the inner sidelobes were then about $5 \%$ of the main beam. All other observations were taken with the telescope after resurfacing, when the beamwidth was $12^{\prime} \times 12^{\prime}$ and the extended sidelobes less than $1 \%$. 


\section{The M81 Group}

The M81 group includes the two I0 galaxies M82 and NGC 3077 within $1^{\circ}$ of M81. Roberts (1972) has already shown that there is an extensive envelope of neutral hydrogen surrounding M81 which appears to form a bridge to M82. In the present survey, observations were taken every $6^{\prime}$ in both RA and Dec over the whole region in which the aerial temperature was more than $0.2 \mathrm{~K}$ and Figure 1 shows the distribution of hydrogen spectra. Hydrogen is found in an area within a radius of $1.2(70 \mathrm{kpc})$ of the centre of M81.

An obvious feature of the distribution is the extension of neutral hydrogen beyond

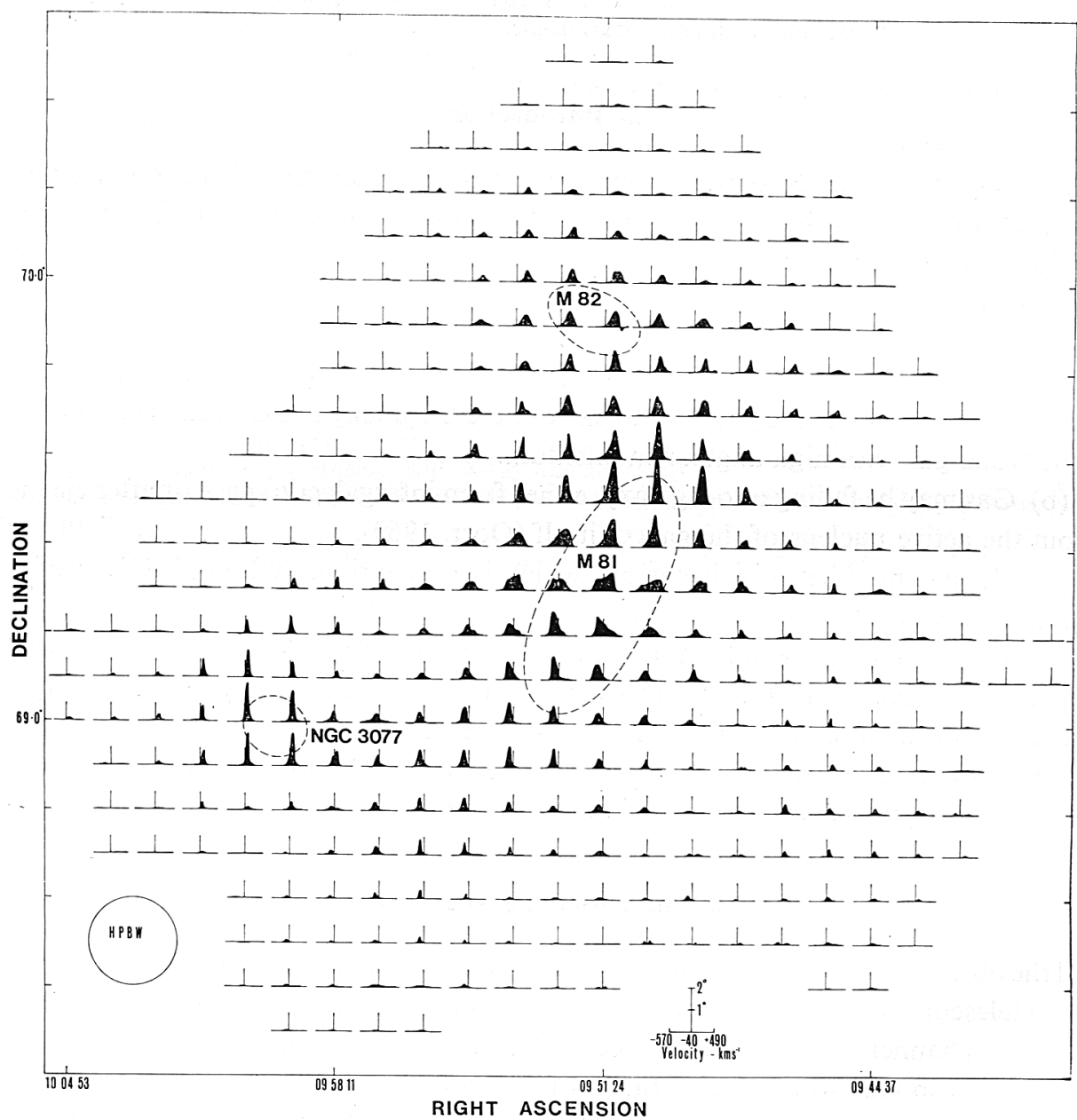

Fig. 1. Neutral hydrogen spectra taken in the M81/M82/NGC 3077 group. The central velocity of each spectrum is $-40 \mathrm{~km} \mathrm{~s}^{-1}$ relative to the Sun; each spectrum extends $\pm 530 \mathrm{~km} \mathrm{~s}^{-1}$. The survey was continued in each direction until the signal fell to below $0.2 \mathrm{~K}$ in brightness temperature. Holmberg optical dimensions for each galaxy are shown by a broken line. 
the Holmberg dimensions which are shown dashed in Figure 1. The hydrogen lies largely in extended envelopes around M81, M82 and NGC 3077 but a neutral hydrogen bridge connects $\mathrm{M} 81$ with its two companion galaxies. A further interesting feature is the presence of a neutral hydrogen companion on the SW side of M81 which can be traced over an area of $0.7 \times 0.5$ situated $0.7 \mathrm{SW}$ of the centre of $\mathrm{M} 81$. The gas in this companion is at a positive velocity relative to that in adjacent areas, which is rotating with $\mathrm{M} 81$.

It is believed that the distributed neutral hydrogen in the M81 group is indeed associated with the group and is not either an artefact of the observing system or foreground galactic emission. Emission in sidelobes of the beam would produce broad spectra at a level of a few hundredths of a degree, insignificant in comparison with the signals observed in Figure 1. The emission is very unlikely to be foreground emission since it follows the velocity distribution expected for M81, is centred on M81 and is not seen in comparison areas $2^{\circ}$ away. The $\mathrm{SW}$ companion has a velocity of $+50 \mathrm{~km} \mathrm{~s}^{-1}$ which is an unlikely velocity in this part of the sky $\left(l=142^{\circ} 1, b=40.9\right)$.

The velocity field of the neutral hydrogen is significant. The bulk of the gas is at velocities which might be expected on the basis of a plausible rotation curve for M81; the gas near the NW major axis is at positive velocities and that near the SE major axis is at negative velocities relative to the centre. Thus, apart from the gas near NGC 3077 and the SW hydrogen companion, it appears to be co-rotating with M81 and is nearly in the same plane as M81.

A preliminary analysis of the data indicates that there is about twice as much neutral hydrogen outside the Holmberg dimensions of the three galaxies as is inside. All the bright neutral hydrogen emission of M81 mapped with the Westerbork synthesis telescope lies inside the Holmberg dimensions.

The present observations give a clear picture of the dynamics of NGC 3077. Its systemic velocity is $+15 \mathrm{~km} \mathrm{~s}^{-1}$ and its major axis lies at p.a. $=60^{\circ}$. The neutral hydrogen diameter is $14^{\prime}$ arc (as defined by the line of centroids of the iso-velocity maps of its emission) which is larger than the Holmberg dimensions of $8.8 \times 8.0$ arc.

The SW neutral hydrogen companion has a neutral hydrogen mass of $\sim 5 \times 10^{7} M_{\odot}$ if it is situated at the distance of M.81 (3.3 Mpc) and, with its observed velocity spread $\left(\sigma \approx 40 \mathrm{~km} \mathrm{~s}^{-1}\right)$ and radius $(4.3 \mathrm{kpc})$, does not appear to be gravitationally stable: a mass of $\sim 1 \times 10^{9} M_{\odot}$ would be required to close such a system. It may be that the velocity dispersion is produced in localized knots which are small enough to be stable, and the whole system is rotating at some lower velocity.

The neutral hydrogen data are not alone in revealing matter outside the Holmberg diameters of the three objects. Two optical companions are seen in the close vicinity of $\mathrm{M} 81$, one $10^{\prime}$ to the east, close to the minor axis, and another $30^{\prime}$ to the south. Both lie within the neutral hydrogen envelope of M81. Arp (1965) has also shown the presence of faint optical emission beyond the Holmberg dimensions of M81.

\section{M31}

In the course of a study of the early-type companions of M31 a neutral hydrogen 
object was discovered which may be a companion of $\mathrm{M} 31$. Its centre is at RA= $=00^{\mathrm{h}} 35^{\mathrm{m}} 10^{\mathrm{s}}, \mathrm{Dec}=42^{\circ} 07^{\prime}(1950)$ which places it 1.5 from the centre and close to the minor axis of M31. It has diameters at half-intensity of $\sim 0.6 \times 0.4$, with a major axis approximately north-south. The cloud has a maximum aerial temperature of $0.5 \mathrm{~K}$ and a mean velocity of $-451 \mathrm{~km} \mathrm{~s}^{-1}$ relative to the Sun; for comparison M31 has a systemic velocity of $\sim-300 \mathrm{~km} \mathrm{~s}^{-1}$. The full width at half-maximum intensity of the spectrum is $22 \mathrm{~km} \mathrm{~s}^{-1}$ which corresponds to a velocity dispersion of $10 \mathrm{~km} \mathrm{~s}^{-1}$.

The neutral hydrogen line integral through the centre of the cloud is $3 \times 10^{19} \mathrm{~cm}^{-2}$, implying a neutral hydrogen mass of $8 \times 10^{6} M_{\odot}$ if the distance is that of M31. With the value of velocity dispersion and diameter given above, this mass will stabilize the cloud gravitationally.

All the evidence is consistent with the hypothesis that this cloud is a gravitationally bound companion of M31. Its velocity and position would allow it to be in a bound orbit. Alternative explanations do not appear attractive. It may be thought that it could be a high velocity cloud (HVC), but its velocity of $-451 \mathrm{~km} \mathrm{~s}^{-1}$ would be the most extreme of any known. Another possibility is that it is part of the Magellanic Stream (this volume p. 367), but again this seems unlikely since (a) the nearest part of the stream lies $\sim 30^{\circ}$ away and (b) the M31 cloud lies $20^{\circ}$ off the projected axis of the stream.

\section{M33}

A series of neutral hydrogen spectra were taken around the outside edge of the aperture synthesis map of M33 made by Wright et al. (1972). Neutral hydrogen had already been shown to exist outside this area by de Jager and Davies (1971), Gordon (1971) and Wright (1973). The present observations (Figure 2) cover an even wider area and are at a higher sensitivity than those published previously. Neutral hydrogen with $T_{\mathrm{b}} \geqslant 0.2$ was found in all positions sampled within $1^{\circ}$ of the centre of M33, but at more than 1.3 from the centre $T_{\mathrm{b}}<0.1 \mathrm{~K}$. The neutral hydrogen in the outer regions is strongest at two positions $\left(\mathrm{RA}=0.0, \mathrm{mec}=-1.0 ; \mathrm{RA}=-3^{\mathrm{m}} \cdot 0, \mathrm{Dec}=+0.7\right)$ which lie within the tilted areas of M33 (de Jager and Davies, 1971).

The velocity of the gas in the vicinity of M33 is close to that which would be expected from an extrapolation of the isovelocity lines of the emission in the inner regions. Consequently it is concluded that this material is rotating with M33 and is a tenuous outer extension of the disk. The neutral hydrogen line integrals of the profiles more than 0.3 from the outer contour shown in Figure 2 range from 2 to $7 \times 10^{19} \mathrm{~cm}^{-2}$. These correspond to a range of 0.02 to $0.06 \mathrm{~cm}^{-3}$ for the density if the gas is concentrated in a disk 200 pc thick.

\section{IC 342}

IC 342 is an Sc galaxy lying close to the galactic plane $(l=138.2, b=10.6)$ where it is heavily obscured by galactic dust. Neutral hydrogen was known to exist outside the optical extent of $\sim 40^{\prime}$ (corrected for $2.2 \mathrm{mag}$ obscuration) as given by Rogstad et al. 


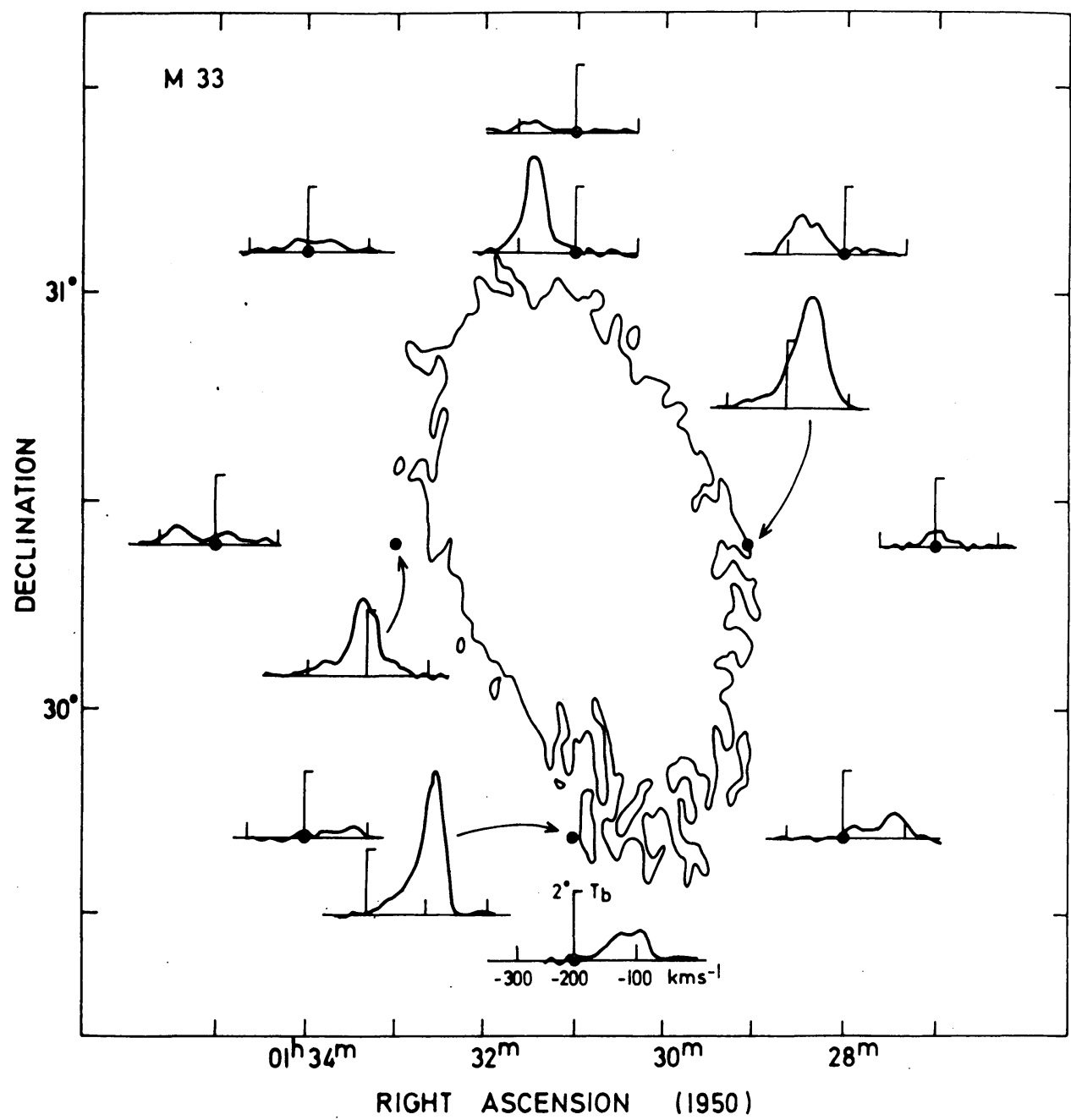

Fig. 2. Neutral hydrogen spectra taken in the vicinity of M33 with an angular resolution of $12^{\prime} \times 12^{\prime}$. The continuous contour is the outer contour $\left(5 \times 10^{20} \mathrm{~cm}^{-2}\right)$ of the aperture synthesis map of Wright et al. (1972). The vertical axis on each spectrum is drawn at a velocity of $-200 \mathrm{~km} \mathrm{~s}^{-1}$ relative to the Sun, and the location of the beam centre is shown by a filled circle.

(1973). For example the aperture synthesis map published by Rogstad et al. shows neutral hydrogen over an area $50^{\prime} \times 40^{\prime}$.

Observations using the $250 \mathrm{ft}$ radiotelescope show extensive neutral hydrogen emission outside the confines of the aperture synthesis map, particularly on the northwestern side. The situation is illustrated in Figure 3. Heavy lines show the loci of the maxima seen in the maps at adjacent velocities. An inner circle follows the region of strongest emission. The outer emission on the NW side lies $\sim 25^{\prime}$ from the centre. A single velocity map illustrates the presence of both the inner emission and the gas 


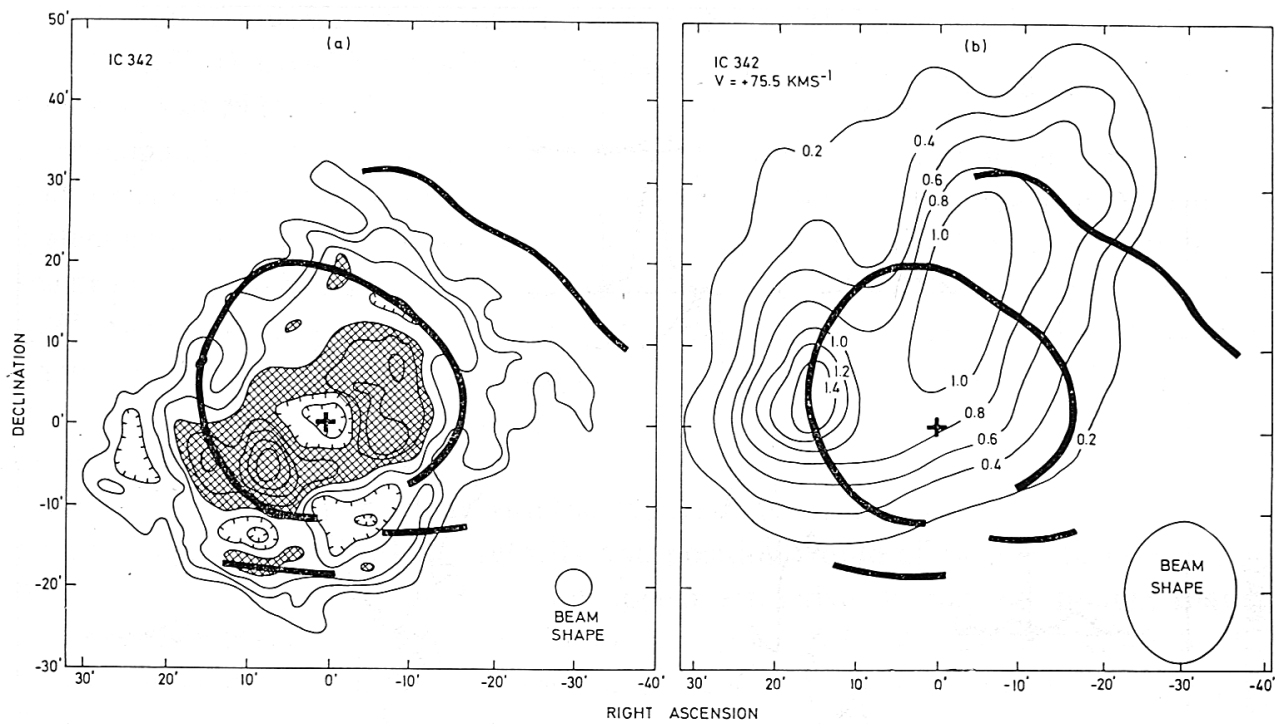

Fig. 3. Neutral hydrogen in IC 342. (a) the light contours are the aperture synthesis map of Rogstad et al. (1973) and the heavy lines are the loci of the centroids of isovelocity maps taken with a $13^{\prime} \times 17^{\prime}$ arc beam. Neutral hydrogen will extend beyond these loci. (b) one constant velocity map of IC 342 superposed on the loci of centroids as described in (a). This illustrates the extent of the emission on the NW side of IC 342 .

on the NW side. Evidently this outer gas is in a distributed layer rather than in narrow bright arms or else it would have been seen in the aperture synthesis maps. Its brightness temperature averaged over a $13^{\prime} \times 17^{\prime}$ beam is about half that of the neutral hydrogen in the inner area.

\section{M51 (NGC 5194/5)}

A survey of M51 has been made with the Mark IA radio telescope. Although the telescope beamwidth $\left(12^{\prime} \times 12^{\prime}\right)$ is comparable with the optical dimensions of the galaxy $\left(8.9 \times 7^{\prime} .4\right)$ clear evidence was found for an extended neutral hydrogen component. This can best be seen on the SE side where the gas extends furthest from the H I outline provided by the aperture synthesis map (Figure 4). The asymmetry of the distribution is marked. An analysis of the observed spectra indicates that neutral hydrogen extends $15^{\prime}$ from the centre of M51 at p.a. $\sim 120^{\circ}$. At this distance the mean surface density is $\sim 2 \times 10^{19} \mathrm{~cm}^{-2}$. As in the case of IC 342 , the gas in the outer regions must be in a distributed form rather than in spiral arms or else it should have been seen in the aperture synthesis map.

In the case of M51, gas is detected out to twice the Holmberg diameter $(8: 9 \times 7: 4)$. The aperture synthesis dimensions given by Weliachew and Gottesman (1973) are comparable with the Holmberg dimensions. Long exposure photographs of M51 (Arp 1966) show optical luminosity out to $\sim 1.5$ times the Holmberg dimensions. The neutral hydrogen in the outer envelope could well have been moved there 


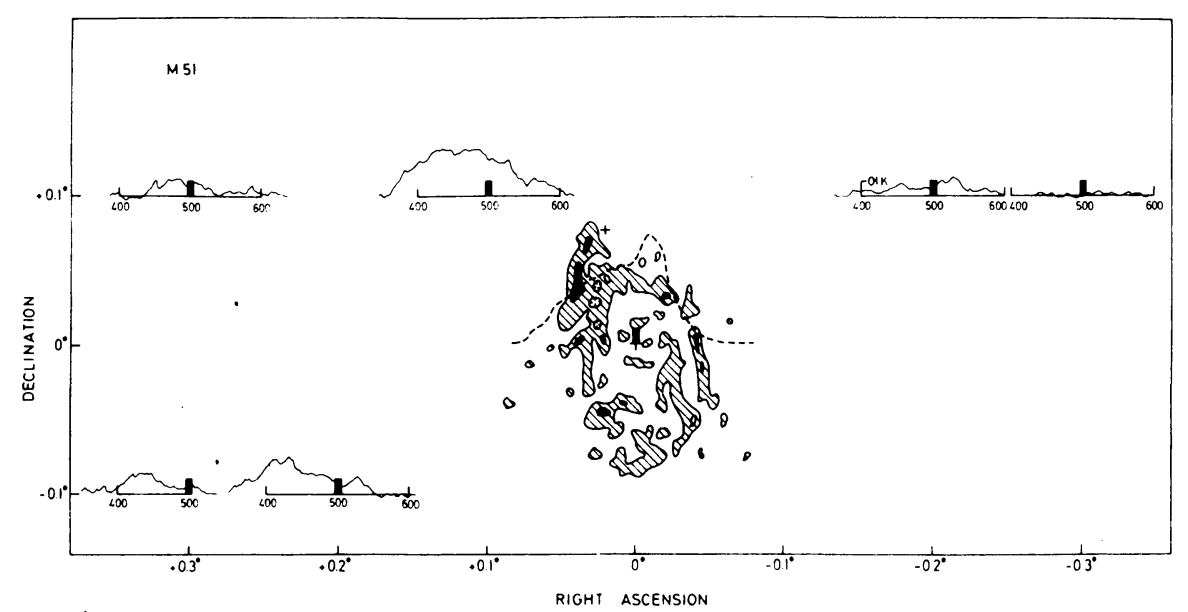

Fig. 4. Neutral hydrogen spectra taken with a resolution of $12^{\prime} \times 12^{\prime}$ in the vicinity of M51. The base of the filled rectangle represents the position of the beam centre for each spectrum and the height represents an aerial temperature of $0.1 \mathrm{~K}$. The neutral hydrogen map taken with the Westerbork synthesis radio telescope is shown for comparison. Emission extends further to the east of M51 than to the west.

in the tidal interaction of NGC 5194 and 5195 as discussed by Toomre and Toomre (1972).

\section{The Magellanic Clouds}

Neutral hydrogen has been known for a long time to exist between the two Magsllanic Clouds. No stars or dust are found in these regions (Hindman et al., 1963). Th2 recent observations by Mathewson, Cleary, and Murray (this volume, p. 367) have shown the existence of substantial amounts of neutral hydrogen extending in streamers from the Magellanic Clouds over large arcs in the sky. They can be traced from $l=90^{\circ}$, $b=-30^{\circ}$, past the south galactic pole to the vicinity of the Magellanic Clouds then on to $l=310^{\circ}, b=0^{\circ}$. The geometry of these streamers and their velocity distribution argue strongly that they are associated with the Magellanic Clouds and have most likely been drawn from the Clouds during a recent encounter with the Galaxy.

It is clear that neutral hydrogen is widely spread throughout the Magellanic CloudMilky Way system. The streamers extend for at least $50 \mathrm{kpc}$ and possibly even further from the Magellanic Clouds. Although the greater part of this gas is in the streamers, an appreciable fraction is also in isolated clouds. A further contribution is in the region lying between the clouds. Figure 5 shows the velocity distribution in a declination cut through the inter-Cloud region at $\mathrm{RA}=03^{\mathrm{h}} 20^{\mathrm{m}}$, to be published by Davies, Murray, Mathewson and Cleary. This emission is typical of the area between the two clouds with a surface density of $\sim 2 \times 10^{20} \mathrm{~cm}^{-2}$.

\section{Conclusions}

Evidence has been presented for a widespread distribution of neutral hydrogen around 


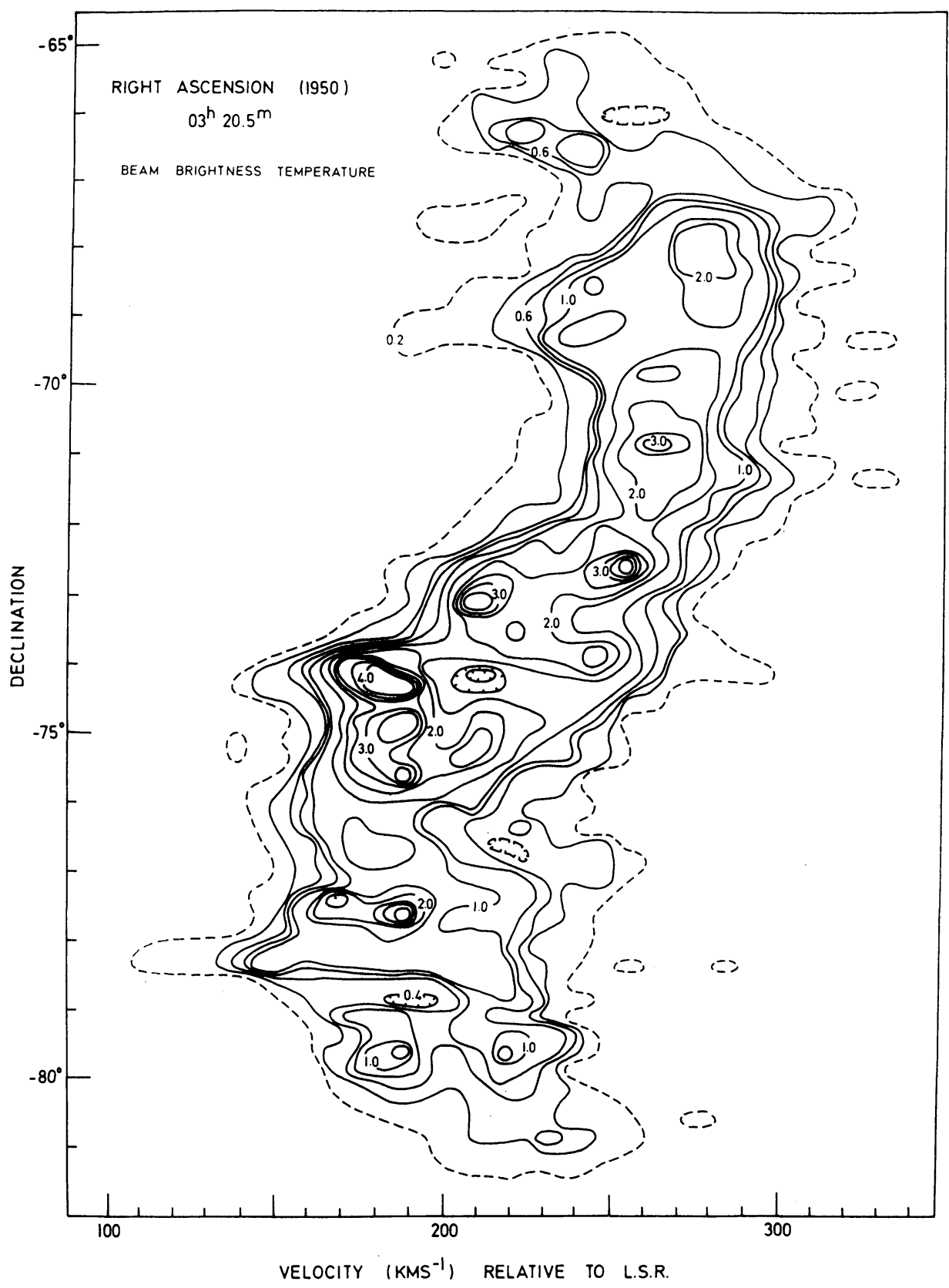

Fig. 5. A declination-velocity diagram taken at Right Ascension $03^{\mathrm{h}} 20^{\mathrm{m}}$ (1973.5); this is a line through the region between the two Magellanic Clouds. The diagram shows the extent of the bright neutral hydrogen emission. The systematic velocity trend with declination can also be seen. 
some galaxies. In all cases where aperture synthesis maps have been made of these galaxies, the neutral hydrogen is found to extend further in the pencil-beam observations, which are more sensitive to extended features. This implies that the gas is distributed in broad features or in a more or less uniform layer outside the main spiral structure in these galaxies. The outer brightness levels in the pencil-beam surveys correspond to neutral hydrogen surface-integrals of $\sim 2 \times 10^{19} \mathrm{~cm}^{-2}$.

In several cases the extended neutral hydrogen envelope may be causally related to the tidal interaction which is occurring between two galaxies at the present time or in the recent past. Galaxies in this category are M33 (see, for example, de Jager and Davies, 1971), the Milky Way-Magellanic Cloud system and the M51 (NGC 5194/95) system. The M81 system could conceivably be of this type but no strong evidence for a gravitational disturbance of the optical objects is found. It is not clear in detail how the gas could be perturbed into the outer parts of these galaxies, although as a general principle the material further from the centre of a subject galaxy is preferentially disturbed in a tidal interaction. Thus any material outside the main spiral structure will show more tidal distortion than the spiral arms themselves. Since the ratio of gas to star mass increases towards the outside edge of galaxies, the gas is likely to show the most severe tidal distortion.

The neutral hydrogen in the M81 system could have had quite a different history. It may never have been closely bound to a particular galaxy but may be orbiting in the general gravitational field of the cluster. The observations suggest that the gas is in a disk-like layer, since the velocity widths of the profiles are small and do not allow a large velocity component out of the plane of rotation of M81. Furthermore the mean velocity of the gas in each spectrum is comparable that with expected for galactic rotation.

\section{Acknowledgements}

I acknowledge the part played by M. W. Bright and R. J. Stephenson in obtaining and analyzing the data for IC 342 and the M81 group. M. N. Cleary, D. S. Mathewson and J. D. Murray collaborated with the author in observations of the Magellanic Cloud system with the Parkes $210 \mathrm{ft}$ radiotelescope.

\section{References}

Arp, H.: 1965, Science 148, 363.

Arp, H.: 1966, Atlas of Peculiar Galaxies, California Institute of Technology, Pasadena, U.S.A. de Jager, G. and Davies, R. D.: 1971, Monthly Notices Roy. Astron. Soc. 153, 9.

Gordon, K. J.: 1971, Astrophys. J. 169, 235.

Hindman, J. V., Kerr, F. J., and McGee, R. X.: 1963, Australian J. Phys. 16, 570.

Holmberg, E.: 1958, Lund. Obs. Medd., Ser. II, No. 136.

Oort, J. H.: 1969, Nature 224, 1158.

Roberts, M. S.: 1972, in D. S. Evans (ed.), 'External Galaxies and Quasi-Stellar Objects', IAU Symp. 44, 12.

Rogstad, D. H., Shostak, G. S., and Rots, A. H.: 1973, Astron. Astrophys. 22, 111.

Toomre, A. and Toomre, J.: 1972, Astrophys. J. 178, 623.

Weliachew, L. and Gottesman, S. T.: 1973, Astron. Astrophys. 24, 59.

Wright, M. C. H., Warner, P. J., and Baldwin, J. E.: 1972, Monthly Notices Roy. Astron. Soc. 155. 337. 
Wright, M. C. H.: 1973, Astrophys. J. 179, 453.

\section{DISCUSSION}

Toomre: After this confirmation by Davies of Mort Robert's huge 21-cm disk around M81 and/or M82, those interested in numerology should recall that the next Messier galaxy, M83 = NGC 5236, was itself discovered about five years ago by B. M. Lewis (Proc. Astron. Soc. Australia 1, 104, 1968) to possess a similar and possibly even larger exterior gas disk. Recent work by Rogstad (as yet unpublished) from Owens Valley not only confirms that disk but likewise provides much fascinating detail.

Lewis: How did you determine the systemic velocity of M82?

Davies: As the mean velocity of the 5 profiles centred on M82 and $\pm 6^{\prime}$ on each side.

Lewis: Are there any double-peaked profiles in the field around M82 or NGC 3077 which might distinguish the $\mathrm{H}_{\mathrm{I}}$ which belongs to each galaxy individually?

Davies: Some double peaked profiles were found in the region between M81 and NGC 3077 which help in separating the emission associated with these objects. No doubling was found between M81 and M82.

Allen: We have observed (paper in preparation) the edge-on system NGC 891 in the radio continuum and $\mathrm{H}$ I at $21 \mathrm{~cm}$ with the Westerbork Telescope in order to study the structure at large $r$ and $z$. The angular resolution was $25^{\prime \prime}$, and the optical image of the galaxy is about $12^{\prime}$ long. The radio continuum data show clearly a flattened radio halo extending up to 2.5 (about $4 \mathrm{kpc}$ ) above the plane of the Galaxy along the rotation axis, and less extensive in the $r$ direction than the optical image. The $\mathrm{H}$ I distribution is closely confined to the plane of the galaxy and extends slightly beyond the edge of the optical image in the $r$ direction. If we were to view this galaxy more face-on, the size of the optical image would be smaller by a factor of about 1.5 , and high-sensitivity $\mathrm{H}$ I observations would reveal $\mathrm{H}$ i far beyond the optical 'edge' of the Galaxy.

G. de Vaucouleurs: The effect of inclination on apparent diameters of galaxies was discussed at length by Heidmann, Heidmann and de Vaucouleurs (Mem. Roy. Astron. Soc. 75, Parts 1-3, 1971). For the Reference Catalogue diameters: $\log D \simeq \log D$ (face-on) $+0.2 \log (D / d)$; for isophotal diameters at $\mu_{B}=25.0 \mathrm{mag}(\operatorname{arcsec})^{-2}$ the coefficient is more precisely 0.235 .

Heidmann: Bottinelli (Astron. Astrophys. 10, 437, 1971) has shown that, for galaxies of the same morphological type as M81, half of the hydrogen mass is contained within half the Holmberg radius. This result is in between what Allen just quoted for NGC 891 and Davies' value for M81. 